\section{Desafios à coordenação dos cuidados em saúde: estratégias de integração entre níveis assistenciais em grandes centros urbanos}

\author{
Challenges for healthcare coordination: strategies \\ for integrating levels of care in large cities
}

\author{
1 Escola Nacional de Saúde \\ Pública Sergio Arouca, \\ Fundação Oswaldo Cruz, Rio \\ de Janeiro, Brasil. \\ Correspondência \\ P. F. Almeida \\ Escola Nacional de Saúde \\ Pública Sergio Arouca, \\ Fundação Oswaldo Cruz. \\ Av. Brasil 4036, sala 1001, \\ Rio de Janeiro, $R J$ \\ 21040-361, Brasil. \\ patty@ensp.fiocruz.br
}

\begin{abstract}
This article analyzes the development of instruments for coordination between the Family Health Strategy and other levels in the health system, with a focus on measures to promote coordination linked to the "integration among levels of care". The results of case studies in four large cities indicate the presence of mechanism for integration among levels of care, suggesting concern with guaranteeing comprehensive care. The principal strategies identified here were: creation and strengthening of regulatory structures in the Municipal Health Secretariats and family health units with decentralization of roles to the local level, organization of flows, electronic patient charts, and expansion in the supply of specialized services at the municipal level. However, lack of integration among different providers, insufficient formal flows for hospital care, and absence of policies for medium complexity care were detected as barriers to the guarantee of comprehensive care, making the network's integration incomplete.
\end{abstract}

Primary Health Care; Systems Integration; Health Services
Patty Fidelis de Almeida 1

Lígia Giovanella 1

Maria Helena Magalhães de Mendonça 1

Sarah Escorel 1

Discussões e problemas vinculados à coordenação dos cuidados em saúde, fragmentação da rede assistencial, ausência de comunicação entre prestadores não constituem temas novos nas discussões sobre a organização dos sistemas de saúde. No entanto, mudanças recentes em relação às demandas e necessidades da população com o crescimento da prevalência de doenças crônicas, que exigem maior contato com os serviços de saúde em um contexto de pressão por otimização das relações de custo-eficiência, tornaram premente a busca de soluções 1,2,3. Na União Européia, desde a década de 1990, reformas pró-coordenação vêm sendo implementadas, sobretudo voltadas ao fortalecimento do primeiro nível de atenção 4,5.

Os ritmos de implantação deste conjunto de reformas têm sido diferenciados entre os países a depender do modelo de proteção social 2,5. Na região da América Latina ainda são incipientes reformas centradas na coordenação dos cuidados, o que se reflete no reduzido número de estudos que tratam deste tema. Segundo Conill \& Fausto ${ }^{5}$, a histórica segmentação e fragmentação que caracterizou a maior parte dos sistemas de saúde latino-americanos, com oferta de Atenção Primária à Saúde seletiva, fragilizaram aspectos como a constituição de redes integradas. Ainda assim, no contexto do recente movimento de valorização e defesa da concepção abrangente de Atenção Primária à Saúde parecem ser promissoras iniciativas pró-coordenação. 
De acordo com as autoras, nesses países, embora com diferenciados graus de desenvolvimento, as principais estratégias para a integração dos sistemas de saúde estão relacionadas à territorialização dos serviços e adscrição de clientela, informatização de histórias clínicas, centrais de marcação de consultas, equipes de especialistas como apoio para a Atenção Primária à Saúde, criação de redes com gestão regional e/ou municipal, entre outras 5 .

A coordenação entre níveis assistenciais pode ser definida como a articulação entre os diversos serviços e ações de saúde relacionada à determinada intervenção de forma que, independentemente do local onde sejam prestados, estejam sincronizados e voltados ao alcance de um objetivo comum ${ }^{6}$. Nesse sentido, reflete-se na existência de uma rede integrada, desde a Atenção Primária à Saúde aos prestadores de maior densidade tecnológica, de modo que distintas intervenções do cuidado sejam percebidas e experienciadas pelo usuário de forma contínua, adequada às suas necessidades de atenção em saúde e compatível com as suas expectativas pessoais 7 . Logo, a coordenação assistencial seria um atributo organizacional dos serviços de saúde que se traduz na percepção de continuidade dos cuidados na perspectiva do usuário.

Hofmarcher et al. 3 , em estudo realizado com policy makers dos países da Organização para a Cooperação e Desenvolvimento Econômico (OECD) e revisão da literatura, identificaram quatro áreas nas quais processos de reforma podem potencializar a capacidade dos sistemas de saúde em melhorar a coordenação entre níveis assistenciais. O primeiro grupo sugere que a coordenação pode ser facilitada pelo aprimoramento da coleta e disseminação das informações sobre pacientes e prestadores e pela ampla utilização das Tecnologias de Informação e Comunicação (TIC). O segundo conjunto de medidas pró-coordenação vincula-se a mudanças necessárias no primeiro nível de atenção para atender às demandas colocadas pelas doenças crônicas. Um terceiro grupo de intervenções com potencial impacto sobre a coordenação dos cuidados refere-se à revisão dos padrões de alocação de recursos no setor ambulatorial dado o progressivo deslocamento de procedimentos da atenção hospitalar para este nível. Por fim, o estudo empreendido pelos autores destaca que a capacidade de coordenação é fortemente afetada pela falta de integração entre os níveis assistenciais e pela relação entre os prestadores, especialmente em função da existência de barreiras que dificultam o trânsito no interior do sistema de saúde.
O presente artigo analisa o desenvolvimento de estratégias e instrumentos de coordenação desde a Estratégia Saúde da Família (ESF) aos demais níveis do sistema de saúde com foco no conjunto de medidas pró-coordenação vinculado à "integração entre níveis assistenciais". Segundo Hartz \& Contandriopoulos 8 (p. 332), “ $a$ integração propriamente dita dos cuidados consiste em uma coordenação durável das práticas clínicas destinadas a alguém que sofre com problemas de saúde, para assegurar a continuidade e a globalidade dos serviços requeridos de diferentes profissionais e organizações, articuladas no tempo e no espaço, conforme os conhecimentos disponíveis". Assim, para efetivar a garantia do cuidado em saúde, torna-se fundamental a constituição de redes integradas, cuja construção reconhece necessariamente a interdependência e, muitas vezes, conflitos entre atores sociais e organizações distintas em situações de poder compartido, visto que nenhuma destas instâncias dispõe da totalidade dos recursos.

Além disso, parte-se do pressuposto de que a integração entre níveis assistenciais é um dos componentes para a oferta de Atenção Primária à Saúde abrangente e distinta da concepção focalizada e seletiva, com cesta restrita e freqüentemente de baixa qualidade, dirigida a populações pobres 9. Conforme destaca Hartz \& Contandriopoulos 8, a efetivação da imagem ideal de um "sistema sem muros" nos quais se eliminariam barreiras de acesso entre os diversos pontos de atenção interligados por corredores virtuais, embora desfrute de relativo consenso em relação aos seus princípios, ainda constitui importante desafio aos sistemas de saúde, demandando avaliações para sua efetiva implementação.

\section{Metodologia}

Este artigo apresenta parte dos resultados de uma pesquisa realizada no ano de 2008, denominada Estudos de Caso sobre a Implementação da Estratégia Saúde da Família em Grandes Centros Urbanos 10 , financiada pelo Departamento de Atenção Básica, Ministério da Saúde. Os municípios para os estudos de caso foram selecionados de forma intencional de modo a escolher experiências consolidadas de implantação da ESF, sendo os casos Aracaju (Sergipe), Belo Horizonte (Minas Gerais), Florianópolis (Santa Catarina) e Vitória (Espírito Santo).

Neste artigo são analisados os resultados referentes à dimensão "integração entre níveis assistenciais" que compuseram um dos eixos principais do estudo. Ênfase foi conferida à relação entre ESF e atenção especializada que en- 
globou serviços de apoio à diagnose e terapia e consultas. Para compreender esta dimensão e orientar a operacionalização do conceito, foram definidas categorias de análise, variáveis e indicadores, com base em revisão de literatura $1,2,3,5,6,7,11,12$ e, sobretudo, estudos de avaliação da ESF 13,14,15,16,17,18,19,20,21 descritos na Tabela 1 .

Os estudos de caso foram desenvolvidos pela utilização de diversos métodos, técnicas, fontes de informação e perspectivas. A descrição e análise das estratégias de integração da rede assistencial empreendidas pela gestão municipal de saúde foram realizadas com base em entrevistas semi-estruturadas com Secretários Municipais de Saúde, Coordenadores de Atenção Básica/ ESF, gerentes vinculados à Regulação, Controle e Avaliação, à Vigilância em Saúde, gerentes de unidades básicas de saúde (UBS), além de representantes de entidades profissionais médicas e de enfermagem. Foram realizadas 54 entrevistas para análise desta dimensão.

As experiências e percepções dos profissionais de saúde sobre coordenação entre níveis assistenciais foram examinadas com base em questionários auto-aplicáveis a amostras de médicos e enfermeiros. Um inquérito de base domiciliar com aplicação de questionários fechados a amostras de famílias cadastradas permitiu conhecer experiências e avaliação dos usuários em relação ao acesso e utilização de serviços de atenção especializada. O informante familiar foi o chefe da família ou cônjuge, entrevistado no domicílio. O plano de amostragem foi do tipo conglomerado em três estágios de seleção: equipe de saúde da família como unidade primária de amostragem, agente comunitário de saúde (ACS) como unidade secundária de amostragem e a família cadastrada como unidade elementar. O tamanho da amostra foi calculado para estimar proporções maiores ou iguais a 0,1 com erro relativo máximo de $30 \%$ (ou seja, erro absoluto máximo de 3\%). Considerando o efeito de conglomeração para seleção de cinco famílias para cada ACS selecionado, obtido na pesquisa Saúde da Família: Avaliação da Implementação em Dez Grandes Centros Urbanos 15, realizada em 20012002, chegou-se a um tamanho de amostra de 768 famílias por município, que dividido por cinco (famílias por ACS) indicou o número de ACS e de ESF a selecionar, tomando por base a seleção de dois ACS por ESF. Ao todo, foram entrevistadas 3.312 famílias (Aracaju 800, Belo Horizonte 900, Florianópolis 790, Vitória 822).

Em decorrência desse cálculo, todas as ESF de Florianópolis (79) e de Vitória (53) foram incluídas na amostra, mas em Vitória foi necessário que se selecionasse três ACS por ESF. Foram selecionadas, com eqüiprobabilidade, 80 das 127
ESF de Aracaju e 90 das 469 ESF de Belo Horizonte. Assim, em Florianópolis e Vitória foi realizado censo de médicos e enfermeiros. Os pesos amostrais foram calculados pelo inverso das probabilidades de inclusão na amostra. Foram entrevistados 224 médicos e 261 enfermeiros nos quatro municípios.

O trabalho de campo foi realizado entre os meses de maio a setembro de 2008. Todos os dados primários resultantes dos estudos transversais foram codificados e computados no programa CSPRO versão 3.3 (U.S. Census Bureau; http://www.census.gov/ipc/www/cspro) e distribuídos em freqüências simples por município, tipo de informante e variável. As entrevistas semi-estruturadas foram gravadas, transcritas e agrupadas por categorias de análise e variáveis. Tanto os dados quantitativos como qualitativos passaram por análise de conteúdo vertical, considerando-se município, categorias e informantes-chave e, posteriormente, por leitura horizontal, comparando-se as perspectivas dos diversos atores envolvidos bem como dos quatro casos analisados.

O estudo foi aprovado pelo Comitê de Ética em Pesquisa da Escola Nacional de Saúde Pública da Fundação Oswaldo Cruz e dos municípios estudados, tendo cumprido todas as exigências estabelecidas pela Resolução $n^{\circ}$. 196/96 do Conselho Nacional de Saúde.

\section{Resultados}

A apresentação dos resultados está estruturada com base nas categorias de análise apresentadas na Tabela 1.

Estrutura organizacional e administrativa do setor de regulação da rede de serviços de saúde

Em Aracaju, a primeira habilitação do sistema de saúde ocorreu no ano de 1998 na condição de Gestão Plena da Atenção Básica conforme Norma Operacional Básica dos SUS (NOB/96), mesmo ano de início da implantação da ESF. Em 2001, assumiu a Gestão Plena do Sistema Municipal e se responsabilizou pela média complexidade e, gradativamente, pelos serviços de alta complexidade. Belo Horizonte, desde o ano de 1994, quando aderiu à Gestão Semi-Plena, iniciou processo de organização de estruturas de controle e avaliação. Em Florianópolis e Vitória, a criação de estruturas de regulação da atenção especializada é recente, tendo sido impulsionada pela expansão de cobertura pela ESF e pela adesão ao Pacto pela Saúde, com assinatura do Ter- 
Categorias de análise, variáveis e indicadores da dimensão "integração entre níveis assistenciais". Quatro grandes centros urbanos, Brasil, 2008.

\begin{tabular}{|c|c|c|}
\hline Categoria de análise & Variáveis & Indicadores \\
\hline \multirow{3}{*}{$\begin{array}{l}\text { Estrutura organizacional } \\
\text { e administrativa do setor } \\
\text { de regulação da rede } \\
\text { de serviços de saúde }\end{array}$} & $\begin{array}{l}\text { Organização de regionais ou distritos de } \\
\text { saúde }\end{array}$ & Existência e número de regionais/Distritos de Saúde \\
\hline & $\begin{array}{c}\text { Estrutura de regulação da Secretaria } \\
\text { Municipal de Saúde }\end{array}$ & $\begin{array}{l}\text { Existência de gerência/coordenação de regulação, avaliação e auditoria da } \\
\text { atenção especializada, hospitalar e da rede de urgência e emergência }\end{array}$ \\
\hline & $\begin{array}{l}\text { Principal propriedade dos prestadores } \\
\text { de serviços de saúde para a atenção } \\
\text { especializada }\end{array}$ & Existência de prestadores públicos municipais e estaduais \\
\hline \multirow[t]{3}{*}{$\begin{array}{l}\text { Instrumentos } \\
\text { de integração dos } \\
\text { serviços de saúde }\end{array}$} & $\begin{array}{l}\text { Sistema municipal de regulação e marcação } \\
\text { de consultas e exames especializados }\end{array}$ & $\begin{array}{l}\text { Existência de central municipal de regulação e marcação de consultas e } \\
\text { exames especializados, percentual de médicos/enfermeiros que informaram } \\
\text { existência de central de marcação de consultas especializadas }\end{array}$ \\
\hline & $\begin{array}{l}\text { Central municipal de regulação e controle de } \\
\text { leitos e internações }\end{array}$ & $\begin{array}{c}\text { Existência de central municipal de regulação } \\
\text { e controle de leitos e internações }\end{array}$ \\
\hline & $\begin{array}{l}\text { Instrumentos de referência e contra- } \\
\text { referência para atenção especializada }\end{array}$ & $\begin{array}{c}\text { Existência de instrumentos de referência e contra-referência, percentual de } \\
\text { famílias cadastradas encaminhadas por profissional da ESF que relataram } \\
\text { receber informações por escrito para entregar ao serviço de referência, } \\
\text { percentual de médicos que fornecem informações por escrito sempre/na } \\
\text { maioria das vezes quando o paciente é referenciado para outros serviços, } \\
\text { percentual de médicos que recebem contra-referência sempre/na maioria } \\
\text { das vezes após consulta do usuário com especialista }\end{array}$ \\
\hline \multirow[t]{5}{*}{$\begin{array}{l}\text { Organização } \\
\text { dos fluxos para } \\
\text { atenção especializada, } \\
\text { hospitalar e de urgência/ } \\
\text { emergência }\end{array}$} & $\begin{array}{l}\text { Percurso mais comum do paciente para } \\
\text { acesso à atenção especializada }\end{array}$ & $\begin{array}{l}\text { Percurso mais comum do paciente desde o atendimento na USF ao } \\
\text { encaminhamento para consulta especializada segundo médicos/enfermeiros } \\
\text { e gestores, principais motivos do encaminhamento pelo médico da ESF nos } \\
\text { últimos } 30 \text { dias segundo famílias cadastradas, principal forma de acesso ao } \\
\text { especialista informada por usuários referenciados nos últimos } 12 \text { meses }\end{array}$ \\
\hline & $\begin{array}{l}\text { Prática sistemática de análise dos } \\
\text { encaminhamentos para serviços } \\
\text { especializados realizados pelos profissionais } \\
\text { da Atenção Primária à Saúde }\end{array}$ & $\begin{array}{c}\text { Existência de prática sistemática de análise dos encaminhamentos para } \\
\text { serviços especializados realizados pelos profissionais da Atenção Primária à } \\
\text { Saúde, percentual de médicos/enfermeiros que referiram existir sempre/na } \\
\text { maioria das vezes supervisão periódica para avaliar a necessidade } \\
\text { de referência aos outros níveis de atenção }\end{array}$ \\
\hline & $\begin{array}{l}\text { Monitoramento das filas de espera para } \\
\text { atenção especializada }\end{array}$ & Existência de monitoramento das filas de espera para atenção especializada \\
\hline & $\begin{array}{l}\text { Fluxo formalizado para acesso à atenção } \\
\text { hospitalar }\end{array}$ & Existência de fluxo formalizado para acesso à atenção hospitalar \\
\hline & $\begin{array}{l}\text { Articulação entre centros de saúde e serviços } \\
\text { de pronto-atendimento }\end{array}$ & $\begin{array}{c}\text { Existência de articulação entre centros de saúde } \\
\text { e serviços de pronto-atendimento }\end{array}$ \\
\hline \multirow{3}{*}{$\begin{array}{l}\text { Acesso e utilização } \\
\text { dos serviços de atenção } \\
\text { especializada e hospitalar }\end{array}$} & $\begin{array}{l}\text { Principal serviço municipal para realização de } \\
\text { consultas/exames especializados }\end{array}$ & Principal serviço para realização de consultas/exames especializados \\
\hline & $\begin{array}{l}\text { Acesso e utilização dos serviços de apoio à } \\
\text { diagnose e terapia e consultas especializadas }\end{array}$ & $\begin{array}{l}\text { Percentual de usuários que relataram necessidade de realização de } \\
\text { exame solicitado pelo médico da ESF nos últimos } 12 \text { meses, percentual } \\
\text { de médicos que conseguem realizar sempre/na maioria das vezes } \\
\text { agendamento para serviços de atenção especializada e de apoio à diagnose } \\
\text { e terapia, percentual de usuários referenciados nos últimos } 12 \text { meses pelo } \\
\text { médico da ESF que conseguiram realizar os exames solicitados, tempo } \\
\text { de espera pelo atendimento com o especialista estimado por usuários } \\
\text { referenciados, tempo médio de espera para consultas especializadas e para } \\
\text { realização de exames de radiologia e ultra-som estimado por médicos/ } \\
\text { enfermeiros, tempo de espera para realização dos exames solicitados pelo } \\
\text { médico da ESF e recebimento dos resultados } \\
\text { estimado por usuários referenciados }\end{array}$ \\
\hline & $\begin{array}{l}\text { Acesso e utilização dos serviços de atenção } \\
\text { hospitalar }\end{array}$ & $\begin{array}{l}\text { Percentual de médicos que conseguem realizar sempre/na maioria das } \\
\text { vezes agendamento para maternidade, tempo médio de espera para } \\
\text { internação para cirurgia eletiva estimado por médicos/enfermeiros }\end{array}$ \\
\hline \multirow{2}{*}{$\begin{array}{l}\text { Instrumentos } \\
\text { para continuidade } \\
\text { informacional }\end{array}$} & Prontuários eletrônicos & $\begin{array}{c}\text { Existência de prontuário eletrônico, percentual de médicos que referiram } \\
\text { fazer registros no prontuário após cada consulta }\end{array}$ \\
\hline & Protocolos clínicos & Implantação de protocolos clínicos \\
\hline
\end{tabular}

ESF: Estratégia Saúde da Família; USF: Unidade de Saúde da Família.

Fonte: Giovanella et al. 10. 
mo de Compromisso de Gestão no ano de 2007, que determinou responsabilidades pela média complexidade.

Nos municípios estudados, observou-se presença de diferentes prestadores de atenção especializada. Em Aracaju e Belo Horizonte, a maioria da oferta localiza-se em unidades próprias municipais. Em Vitória, volume considerável de exames ainda se encontra sob gestão do estado, sendo esta rede maior que a municipal. Em Florianópolis, segundo gerentes responsáveis pela regulação, a maior parte da oferta de atenção especializada já está sendo prestada por unidades municipais (Tabela 2). Nestes dois casos, as cotas para os serviços sob gestão estadual são definidas pela Programação Pactuada Integrada (PPI) e controladas por centrais de regulação estaduais.

A compra de consultas e exames da rede privada, particularmente nas especialidades com maior fila de espera, foi outra estratégia para garantia de atenção especializada nos quatro casos. Neste sentido, foi relatado que os valores pagos pela tabela SUS, considerados insuficientes pelos prestadores, e a ausência de políticas de média complexidade pelo Ministério da Saúde representam importantes entraves à oferta de atenção integral, exigindo pesados investimentos municipais.

\section{Instrumentos de integração}

dos serviços de saúde

Nos quatro municípios, um dos principais instrumentos para integrar a atenção básica à média complexidade foi a implantação de centrais informatizadas de regulação e marcação de procedimentos desde os centros de saúde. Belo Horizonte, Florianópolis e Vitória optaram pelo SISREG, sistema de regulação on line disponibilizado pelo Departamento de Informática do SUS (DATASUS), para gerenciar e operar centrais de regulação. Aracaju implantou o TAS (Terminal de Atendimento do SUS), que permite a marcação de procedimentos especializados, mas não desempenha funções de regulação (Tabela 2). Os procedimentos oferecidos por meio da PPI ainda não estavam sob regulação dos sistemas municipais. As iniciativas de regulação e integração entre atenção básica e atenção especializada são confirmadas pela grande maioria dos médicos e enfermeiros das equipes de saúde da família que reconhecem a existência de centrais de marcação de consultas e exames (Tabela 3).

Além das centrais informatizadas, os municípios apresentaram estratégias para descentralizar as discussões e a responsabilização pelas ações de regulação para os profissionais da atenção básica. Há uma compreensão de que a primeira instância regulatória é o centro de saúde. Em Belo Horizonte, concomitantemente à implantação do SISREG, foi realizado trabalho de discussão sobre o conceito de regulação assistencial junto aos distritos e unidades básicas. Segundo os gestores e gerentes da Secretaria Municipal de Saúde (SMS), em um contexto de oferta limitada de recursos é necessário estabelecer prioridades até para que se possa garantir a eqüidade. O primeiro a estabelecer critérios de priorização é o centro de saúde, depois o distrito e por fim a SMS: "Existem todas estas instâncias de regulação para garantir o acesso em um tempo oportuno e favorecer o restabelecimento da saúde, da autonomia e garantir a atenção integral" (gestor local de Belo Horizonte). Em Florianópolis e Vitória, tal processo é ainda incipiente, embora, com a implantação do SISREG e organização das listas de espera por unidades de saúde, o processo de descentralização das discussões sobre regulação esteja avançando.

Em Belo Horizonte, todos os leitos, inclusive os privados credenciados ao SUS e os estaduais, são controlados pela central municipal de regulação. Em Florianópolis e Vitória, não há central municipal de regulação de leitos e internações uma vez que os serviços hospitalares são de gestão estadual. Os dois municípios possuem serviços intermediários de pronto-atendimento que estabelecem ligação com a central de marcação de leitos do estado, para os casos de urgência. Aracaju, embora seja Gestor Pleno do Sistema Municipal, também não possui central municipal de regulação de leitos e internações (Tabela 2).

Instrumentos de referência e contra-referência representam importante instrumento para integração dos serviços de saúde. Nos quatro municípios foi relatada a existência de mecanismos formais para referência e contra-referência entre atenção básica e especializada. Belo Horizonte e Florianópolis avançam na informatização das unidades básicas e especializadas com implantação de prontuários eletrônicos. A maior parte dos gestores e gerentes em todas as cidades afirmou que a contra-referência não é uma prática comum, apesar de os fluxos estarem instituídos.

O fornecimento de informações escritas sempre/na maioria das vezes quando o usuário é referenciado a outro serviço de saúde - foi prática confirmada pela maioria dos médicos entrevistados, alcançando percentuais superiores a $90 \%$ em Vitória, Belo Horizonte e Florianópolis. Mais da metade das famílias encaminhadas por profissionais da ESF também afirmou receber informações por escrito para entregar ao especialista, sendo o maior percentual verificado em Vitória (85\%) (Tabela 4). Cabe ressaltar que a implan- 


\section{Tabela 2}

Estratégias e características do processo de integração entre os níveis assistenciais segundo gestores e gerentes. Quatro grandes centros urbanos, Brasil, 2008.

\begin{tabular}{|c|c|c|c|c|}
\hline Variáveis & Aracaju & Belo Horizonte & Florianópolis & Vitória \\
\hline \multicolumn{5}{|l|}{$\begin{array}{l}\text { Estrutura organizacional e administrativa } \\
\text { do setor de regulação } \\
\text { da rede de serviços de saúde }\end{array}$} \\
\hline $\begin{array}{l}\text { Organização de regionais ou } \\
\text { distritos de saúde }\end{array}$ & 8 Regiões de Saúde & 9 Distritos Sanitários & 5 Regionais de Saúde & 6 Regiões de Saúde \\
\hline $\begin{array}{l}\text { Estrutura de regulação da } \\
\text { Secretaria Municipal de Saúde }\end{array}$ & $\begin{array}{l}\text { Núcleo de Controle, } \\
\text { Avaliação, Auditoria e } \\
\text { Regulação }\end{array}$ & $\begin{array}{c}\text { Gerência de Regulação } \\
\text { e Atenção Hospitalar, } \\
\text { de Projetos Especiais, } \\
\text { de Urgência e de Apoio } \\
\text { Diagnóstico }\end{array}$ & $\begin{array}{c}\text { Coordenação } \\
\text { de Regulação e } \\
\text { Coordenação de } \\
\text { Controle, Avaliação e } \\
\text { Auditoria }\end{array}$ & $\begin{array}{c}\text { Gerência de Regulação, } \\
\text { Controle e Avaliação }\end{array}$ \\
\hline $\begin{array}{l}\text { Principal propriedade dos } \\
\text { prestadores de serviços de saúde } \\
\text { para atenção especializada }\end{array}$ & Município & Município & Município & Estado \\
\hline \multicolumn{5}{|l|}{$\begin{array}{l}\text { Instrumentos de integração dos serviços } \\
\text { de saúde }\end{array}$} \\
\hline $\begin{array}{l}\text { Sistema municipal de regulação e } \\
\text { marcação de consultas e exames } \\
\text { especializados }\end{array}$ & TAS & SISREG & SISREG & SISREG \\
\hline $\begin{array}{l}\text { Central municipal de regulação e } \\
\text { controle de leitos e internações }\end{array}$ & Não & $\operatorname{Sim}$ & Não & Não \\
\hline $\begin{array}{l}\text { Instrumentos de referência e } \\
\text { contra-referência para atenção } \\
\text { especializada }\end{array}$ & $\begin{array}{l}\text { Sim (guias para } \\
\text { preenchimento manual) }\end{array}$ & Sim (informatizado) & Sim (informatizado) & $\begin{array}{l}\text { Sim (guias para } \\
\text { preenchimento manual) }\end{array}$ \\
\hline \multicolumn{5}{|l|}{$\begin{array}{l}\text { Organização dos fluxos para atenção } \\
\text { especializada, hospitalar e de urgência/ } \\
\text { emergência }\end{array}$} \\
\hline $\begin{array}{l}\text { Percurso mais comum do } \\
\text { paciente para acesso à atenção } \\
\text { especializada }\end{array}$ & $\begin{array}{l}\text { Encaminhamento dos } \\
\text { centros de saúde }\end{array}$ & $\begin{array}{l}\text { Encaminhamento dos } \\
\text { centros de saúde }\end{array}$ & $\begin{array}{l}\text { Encaminhamento dos } \\
\text { centros de saúde }\end{array}$ & $\begin{array}{l}\text { Encaminhamento dos } \\
\text { centros de saúde }\end{array}$ \\
\hline $\begin{array}{l}\text { Pratica sistemática de análise dos } \\
\text { encaminhamentos para serviços } \\
\text { especializados realizados pelos } \\
\text { profissionais da Atenção Primária } \\
\text { à Saúde }\end{array}$ & Não & Não & Não & Não \\
\hline $\begin{array}{l}\text { Monitoramento das filas de espera } \\
\text { para atenção especializada }\end{array}$ & Parcial & Sim & Sim & Sim \\
\hline $\begin{array}{l}\text { Fluxo formalizado para acesso à } \\
\text { atenção hospitalar }\end{array}$ & Não & Sim & Não & Não \\
\hline $\begin{array}{l}\text { Articulação entre centros de saúde } \\
\text { e serviços de pronto-atendimento }\end{array}$ & Não & Sim & Não & Não \\
\hline \multicolumn{5}{|l|}{$\begin{array}{l}\text { Acesso e utilização dos serviços de } \\
\text { atenção especializada e hospitalar }\end{array}$} \\
\hline $\begin{array}{l}\text { Principal serviço municipal para } \\
\text { realização de consultas/exames } \\
\text { especializados }\end{array}$ & $\begin{array}{c}\text { Centros de } \\
\text { Especialidades Médicas } \\
\text { de Aracaju }\end{array}$ & $\begin{array}{l}\text { Unidades de Referência } \\
\text { Secundária e Centros de } \\
\text { Especialidades Médicas }\end{array}$ & Policlínicas Municipais & $\begin{array}{l}\text { Centro Municipal de } \\
\text { Especialidades }\end{array}$ \\
\hline \multicolumn{5}{|l|}{$\begin{array}{l}\text { Instrumentos para continuidade } \\
\text { informacional }\end{array}$} \\
\hline Prontuários eletrônicos & Não & Sim & Sim & Não \\
\hline Protocolos clínicos & Sim (consolidado) & Sim (consolidado) & $\begin{array}{l}\text { Sim (em fase inicial de } \\
\text { implantação) }\end{array}$ & $\begin{array}{l}\text { Sim (em fase inicial de } \\
\text { implantação) }\end{array}$ \\
\hline
\end{tabular}

SISREG: Sistema de Regulação do SUS; TAS: Terminal de Atendimento do SUS.

Fonte: Giovanella et al. 10. 
Tabela 3

Indicadores de integração entre níveis assistenciais segundo médicos e enfermeiros das Equipes de Saúde da Família. Quatro grandes centros urbanos, Brasil, 2008

\begin{tabular}{|c|c|c|c|c|}
\hline Indicadores & Aracaju * & Belo Horizonte ** & 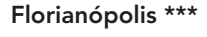 & Vitória \# \\
\hline $\begin{array}{l}\text { Percentual de médicos/enfermeiros que informaram } \\
\text { existência de central de marcação de consultas } \\
\text { especializadas }\end{array}$ & 90,2 & 99,3 & 89,3 & 92,9 \\
\hline $\begin{array}{l}\text { Percentual de médicos que fornecem informações por } \\
\text { escrito sempre/na maioria das vezes quando o paciente } \\
\text { é referenciado para outros serviços }\end{array}$ & 76,8 & 95,8 & 93,4 & 100,0 \\
\hline $\begin{array}{l}\text { Percentual de médicos que recebem a contra-referência } \\
\text { sempre/na maioria das vezes após consulta do usuário com } \\
\text { especialista }\end{array}$ & 5,4 & 2,8 & 11,5 & 11,4 \\
\hline \multicolumn{5}{|l|}{$\begin{array}{l}\text { Percurso mais comum do paciente desde atendimento } \\
\text { na USF ao encaminhamento para consulta especializada } \\
\text { segundo médicos/enfermeiros }\end{array}$} \\
\hline $\begin{array}{l}\text { A consulta é agendada pela USF e a data posteriormente } \\
\text { informada ao paciente }\end{array}$ & 44,3 & 95,9 & 95,4 & 62,4 \\
\hline O paciente sai da USF com a consulta agendada & 23,8 & 1,4 & 0,8 & 12,9 \\
\hline $\begin{array}{l}\text { Percentual de médicos/enfermeiros que referiram existir } \\
\text { sempre/na maioria das vezes supervisão periódica para } \\
\text { avaliar a necessidade de referência a outros níveis de } \\
\text { atenção }\end{array}$ & 26,3 & 25,9 & 19,8 & 11,8 \\
\hline \multicolumn{5}{|l|}{$\begin{array}{l}\text { Percentual de médicos que conseguem realizar sempre/ } \\
\text { na maioria das vezes agendamento para outros serviços }\end{array}$} \\
\hline Serviços de atenção especializada & 42,9 & 80,6 & 47,6 & 71,5 \\
\hline Serviço de apoio diagnóstico e terapia & 37,5 & 55,5 & 42,7 & 42,9 \\
\hline Maternidade & 57,2 & 86,1 & 75,4 & 74,3 \\
\hline \multicolumn{5}{|l|}{ Tempo médio de espera estimado por médicos/enfermeiros } \\
\hline \multicolumn{5}{|l|}{ Consultas especializadas } \\
\hline Em até 1 mês & 23,7 & 13,6 & 13,0 & 41,2 \\
\hline 3 meses e mais & 32,0 & 53,1 & 57,3 & 34,1 \\
\hline \multicolumn{5}{|l|}{ Realização exames de radiologia e ultra-som } \\
\hline Em até 1 mês & 26,3 & 42,1 & 19,8 & 48,6 \\
\hline 3 meses e mais & 40,2 & 27,2 & 51,1 & 18,8 \\
\hline \multicolumn{5}{|l|}{ Internação para cirurgia eletiva } \\
\hline Não sabe informar & 38,5 & 38,8 & 30,5 & 44,7 \\
\hline $\begin{array}{l}\text { Percentual de médicos que referiram fazer registros } \\
\text { no prontuário após cada consulta }\end{array}$ & 100,0 & 100,0 & 100,0 & 100,0 \\
\hline \multicolumn{5}{|l|}{$\begin{array}{l}\text { Principais problemas para integração da rede de serviços } \\
\text { segundo médicos/enfermeiros da ESF }\end{array}$} \\
\hline $\begin{array}{l}\text { Relação entre a USF e a atenção especializada no setor } \\
\text { de cuidados ambulatoriais }\end{array}$ & 60,7 & 46,9 & 55,0 & 54,1 \\
\hline Relação entre USF e emergência & 45,1 & 63,3 & 39,7 & 61,2 \\
\hline $\begin{array}{l}\text { Problemas por causa das listas de espera que impedem } \\
\text { o acesso adequado ao cuidado especializado e } \\
\text { hospitalar }\end{array}$ & 81,1 & 91,8 & 91,6 & 89,4 \\
\hline
\end{tabular}

* 56 médicos e 66 enfermeiros;

** 72 médicos e 75 enfermeiros;

*** 61 médicos e 70 enfermeiros;

\# 35 médicos e 50 enfermeiros.

ESF: Estratégia Saúde da Família; USF: Unidade de Saúde da Família.

Fonte: Giovanella et al. 10. 
Indicadores de acesso e utilização de serviços de atenção especializada segundo famílias cadastradas por Equipes de Saúde da Família. Quatro grandes centros urbanos, Brasil, 2008.

\begin{tabular}{|c|c|c|c|c|}
\hline Indicadores & Aracaju & $\begin{array}{l}\text { Belo } \\
\text { Horizonte }\end{array}$ & Florianópolis & Vitória \\
\hline $\begin{array}{l}\text { Percentual de famílias cadastradas encaminhadas por profissional da ESF que } \\
\text { relataram receber informações por escrito para entregar ao serviço ou especialista } \\
\text { de referência }\end{array}$ & $65,3(n=346)$ & $77,0(n=582)$ & $54,4(n=469)$ & $84,8(n=468)$ \\
\hline $\begin{array}{l}\text { Principais motivos do encaminhamento pelo médico da ESF nos últimos } 30 \text { dias } \\
\text { segundo famílias cadastradas }\end{array}$ & $n=60$ & $n=101$ & $\mathrm{n}=58$ & $n=132$ \\
\hline Consulta médica com especialista & 73,3 & 71,3 & 73,3 & 68,9 \\
\hline Exames & 33,3 & 28,7 & 33,3 & 27,3 \\
\hline Internação ou cirurgia & - & 9,9 & - & 3,8 \\
\hline $\begin{array}{l}\text { Principal forma de acesso ao especialista informada por usuários referenciados } \\
\text { nos últimos } 12 \text { meses }\end{array}$ & $n=303$ & $n=324$ & $n=227$ & $n=330$ \\
\hline A consulta com especialista foi agendada pela USF & 43,2 & 66,7 & 40,1 & 62,7 \\
\hline Procurou por conta própria sem qualquer encaminhamento & 25,7 & 19,4 & 15,0 & 19,4 \\
\hline $\begin{array}{l}\text { Percentual de usuários que relataram necessidade de realização de exame } \\
\text { solicitado pelo médico da ESF nos últimos } 12 \text { meses }\end{array}$ & $57,8(n=470)$ & $52,0(n=583)$ & $51,8(n=336)$ & $53,0(n=491)$ \\
\hline $\begin{array}{l}\text { Percentual de usuários referenciados nos últimos } 12 \text { meses pelo médico da ESF } \\
\text { que conseguiram realizar os exames solicitados }\end{array}$ & $86,0(n=272)$ & $93,1(n=303)$ & $78,7(n=174)$ & $90,0(n=260)$ \\
\hline $\begin{array}{l}\text { Tempo de espera pelo atendimento com o especialista estimado } \\
\text { por usuários referenciados }\end{array}$ & $n=163$ & $n=241$ & $n=162$ & $n=231$ \\
\hline Até 30 dias & 59,5 & 58,1 & 43,9 & 76,5 \\
\hline 3 meses e mais & 9,2 & 17,0 & 16,0 & 10,8 \\
\hline \multicolumn{5}{|l|}{ Tempo de espera estimado por usuários referenciados por médico da ESF } \\
\hline Realização dos exames & $n=234$ & $\mathrm{n}=282$ & $n=137$ & $n=234$ \\
\hline De 0 a 7 dias & 18,0 & 53,6 & 19,0 & 50,4 \\
\hline Em até 30 dias & 68,4 & 89,4 & 57,7 & 90,6 \\
\hline Recebimento dos resultados dos exames & $n=234$ & $n=282$ & $n=137$ & $n=234$ \\
\hline De 0 a 7 dias & 19,2 & 22,3 & 51,8 & 36,3 \\
\hline Em até 30 dias & 76,5 & 91,4 & 84,6 & 93,6 \\
\hline
\end{tabular}

ESF: Estratégia Saúde da Família; USF: Unidade de Saúde da Família.

Fonte: Giovanella et al. 10.

tação dos prontuários eletrônicos em Belo Horizonte e Florianópolis, mesmo parcial, pode ter apresentado influência na diminuição de guias manuais de referência e contra-referência conduzidas pelo usuário. A insuficiência de contrareferência apontada pelos gerentes e gestores foi confirmada pelos baixos percentuais de médicos que afirmaram recebê-la, sempre e na maioria das vezes, variando de $2,8 \%$ em Belo Horizonte a 11,5\% em Florianópolis (Tabela 3).

\section{Organização dos fluxos para atenção especializada, hospitalar e de urgência/emergência}

O percurso mais comum do usuário para acesso à atenção especializada acontece por meio de encaminhamentos dos serviços de atenção bási- ca segundo gestores, gerentes, médicos e enfermeiros entrevistados. Nos municípios estudados, a marcação é feita dentro do centro de saúde com base na referência realizada pelo médico (Tabela 2). Na maior parte das vezes, o pedido é inserido no sistema de acordo com classificação de riscos e tem a data e serviço de referência a ser procurado posteriormente avisado ao usuário. Em Belo Horizonte e Florianópolis, essa opção representou quase a totalidade das respostas (Tabela 3) de médicos e enfermeiros.

Consultas com especialistas foi o principal motivo de encaminhamento para cerca de $70 \%$ das famílias com experiência de atendimento recente pela ESF nos quatro casos (Tabela 4). Segundo usuários referenciados ao especialista nos últimos 12 meses, $67 \%$ e $63 \%$, respectivamente, em Belo Horizonte e Vitória, afirmaram que a 
consulta foi agendada pela Unidade de Saúde da Família (USF), sendo encontrados percentuais em torno de $40 \%$ em Florianópolis e Aracaju. Parte importante dos usuários procurou serviços especializados por conta própria, especialmente em Aracaju (26\%), o que pode indicar barreiras à constituição da porta de entrada pela Atenção Primária à Saúde, organização de fluxos e utilização de planos privados de saúde (Tabela 4).

Não foi referida prática sistemática de análise dos encaminhamentos para a atenção especializada realizada pelas equipes de saúde da família por parte da SMS ou por supervisores (Tabela 2). A existência de supervisão periódica para análise das referências sempre/na maioria das vezes foi referida por $26 \%$ dos médicos e enfermeiros de Aracaju e Belo Horizonte, indicando a presença de iniciativas mais pontuais. Os percentuais encontrados foram menores em Vitória e Florianópolis, municípios que recém assumiram a responsabilidade pela gestão da atenção especializada (Tabela 3 ).

Além de regular o acesso e a marcação de consultas e exames, a implantação do SISREG em três municípios estudados permitiu o monitoramento on line das filas de espera para a atenção especializada, propiciando à equipe de saúde da família acompanhar o percurso do usuário. Com base nos relatórios gerados pelo SISREG, Florianópolis e Vitória desencadearam amplo processo de reavaliação das filas de espera junto aos centros de saúde. Em Aracaju, segundo gerentes e gestores da SMS, o TAS não produz relatórios que permitam monitorar as filas de espera, sendo a percepção sobre os tempos proveniente do controle que cada gerente de centro de saúde faz dos encaminhamentos realizados e de relatórios produzidos pela regulação (Tabela 2).

Em Aracaju, Florianópolis e Vitória não foi referido o estabelecimento de fluxos formalizados para acesso à atenção hospitalar. Para as internações de urgência e emergência, a busca por vagas na rede hospitalar é freqüentemente realizada pelos serviços intermediários de pronto-atendimento. No caso de cirurgias eletivas, mecanismos informais são utilizados para a garantia de internação como contato pessoal entre médicos ou o próprio usuário se responsabiliza pela busca do atendimento. Em Belo Horizonte, a central municipal de regulação de leitos e internações realiza buscas para garantia das internações de urgência tendo como critérios prioridades clínicas e o perfil do prestador. Para os casos de cirurgias eletivas, há uma junta responsável pela análise das Autorizações de Internação Hospitalar (AIH) (Tabela 2), composta por médicos reguladores.

Em Belo Horizonte, as portas de entrada para atenção de urgência e emergência são compos- tas por unidades públicas sob gestão municipal. Neste município, o processo de articulação e definição de fluxos entre centros de saúde e Unidades de Pronto-Atendimento (UPA) foi avaliado de forma positiva pelos gestores. Em Florianópolis, Vitória e Aracaju, não foram mencionados mecanismos de referência e contra-referência entre unidades básicas e pronto-atendimento (Tabela 2). A relação entre USF e os serviços de emergência foi avaliado como um dos principais problemas para integração da rede por alto percentual de médicos e enfermeiros em Vitória (61\%) e em Belo Horizonte (63\%), apesar das iniciativas municipais para estabelecimento de fluxos no último (Tabela 3).

\section{Acesso e utilização dos serviços de atenção} especializada e hospitalar

Outra estratégia identificada para ampliar o acesso à atenção especializada e integração da rede foi a criação, pelos governos municipais de Belo Horizonte e Florianópolis, de serviços territorializados, que funcionam como referência para as unidades de atenção básica de determinado distrito ou regional de saúde. Além do aumento da oferta, a descentralização da atenção secundária vem permitindo maior contato entre generalistas e especialistas, garantia de acesso em tempo mais oportuno e diminuição de barreiras financeiras de acesso. Gestores locais de Florianópolis avaliam que investimentos em média complexidade potencializam a resolubilidade da atenção básica: "É dentro desta lógica que estamos trabalhando, ou seja, ampliar o acesso à média complexidade é também qualificar a atenção primária" (gestor local de Florianópolis). Em Aracaju, cada CEMAR (Centro de Especialidades Médicas de Aracaju) é referência para todos os centros de saúde do município em função das especialidades oferecidas (Tabela 2). Ainda assim, a oferta de atenção especializada foi considerada insuficiente nos quatro municípios.

A descentralização e ampliação da oferta de atenção especializada refletem-se na avaliação de médicos e enfermeiros de forma diferenciada entre os municípios. Em Belo Horizonte (81\%) e Vitória $(72 \%)$ a maioria dos profissionais médicos afirmaram conseguir realizar agendamento para serviços de atenção especializada sempre e na maioria das vezes. Avaliações menos positivas foram encontradas em Aracaju (43\%) e Florianópolis (48\%). As dificuldades são maiores em relação à rede de apoio diagnóstico. Em Aracaju, Florianópolis e Vitória o percentual de profissionais médicos que afirmaram conseguir realizar sempre/na maioria das vezes agendamento para esses serviços ficou em torno de $40 \%$ (Tabela 3 ). 
Mesmo com as dificuldades apontadas, altos percentuais de usuários referenciados nos últimos 12 meses, variando de $93 \%$ em Belo Horizonte a $79 \%$ em Florianópolis afirmaram conseguir realizar os exames solicitados pelos médicos da Equipe de Saúde da Família (Tabela 4).

Em relação aos tempos de espera para atendimento com especialistas, cerca da metade dos médicos e enfermeiros de Florianópolis (57\%) e Belo Horizonte (53\%) estimou ser de três meses e mais. Em Vitória, constatou-se maior agilidade, sendo de até um mês o tempo de espera estimado por $41 \%$ dos profissionais (Tabela 3 ). Entre os usuários que haviam sido referenciados ao especialista, a maioria referiu ter sido atendido em até um mês em três municípios, exceto em Florianópolis (44\%), sendo o maior percentual encontrado em Vitória (77\%) (Tabela 4). Para realização de exames de radiologia e ultra-som, o tempo médio de espera estimado pela maior parte dos médicos e enfermeiros de Aracaju e Florianópolis foi de três meses e mais. Já em Vitória e Belo Horizonte, foi afirmado que tais exames são realizados em até um mês. A avaliação dos usuários indica menores tempos de espera para realização de exames, sendo de até sete dias em Belo Horizonte $(54 \%)$ e Vitória (50\%) para a maioria dos entrevistados. Maior agilidade no recebimento dos resultados foi observada em Florianópolis e Vitória (Tabela 4).

Em Belo Horizonte, em alguns casos o atendimento hospitalar é territorializado como, por exemplo, na atenção à gestante, confirmado pelo alto percentual de médicos (86\%) que afirmaram conseguir realizar sempre/na maioria das vezes agendamento para maternidade (Tabela 3). Em Florianópolis e Vitória, a atenção hospitalar é regulada pelo estado e representa importante limite à oferta e acesso à atenção integral em saúde porque são frágeis os mecanismos de integração entre os serviços municipais, estaduais e federais: "Quando precisa encaminhar um paciente para internação, não há um fluxo estabelecido. Superar essa dificuldade certamente qualificaria e integraria melhor a rede" (gestor local de Florianópolis). Nos quatro municípios, as internações para cirurgias eletivas não se encontravam sob governabilidade dos profissionais de atenção básica, sendo bastante elevado o número de médicos e enfermeiros que não souberam estimar o tempo médio de espera (Tabela 3).

\section{Instrumentos para continuidade informacional}

Belo Horizonte e Florianópolis, no momento da pesquisa, estavam em processo de implantação de prontuários eletrônicos nos centros de saúde e serviços municipais de especialidades. Fazer registros nos prontuários após cada consulta foi uma prática referida pela totalidade dos médicos de família em todas as cidades (Tabela 3).

Os quatro municípios adotam protocolos clínicos em diversas áreas do cuidado que, embora sigam recomendações gerais do Ministério da Saúde, passaram por processos de discussão local e revisões, com participação de profissionais, gestores e entidades representativas (Tabela 2). Em Belo Horizonte, Florianópolis e Vitória, além dos protocolos tradicionais com base nos ciclos de vida e agravos, foram adotados também protocolos para definição de fluxos e regulação da atenção especializada. Em Florianópolis e Vitória, alguns protocolos se encontravam em fase inicial de implantação ou sendo reestruturados para atender às novas configurações da rede e responsabilidades assumidas pelo município.

\section{Discussão}

Estudos apontam que a capacidade dos sistemas de saúde em prestar cuidados mais coordenados ao minimizar barreiras de acesso entre níveis assistenciais, oferecer atenção em saúde mais sincronizada e em tempo oportuno pode ser otimizada pela implantação de mecanismos e estratégias de integração da rede assistencial 2,3,6,8. Além disso, os serviços de Atenção Primária à Saúde, pelos atributos que os caracterizam 22, seriam os mais adequados para assumirem a responsabilidade de coordenar o percurso terapêutico do usuário na maior parte dos episódios de adoecimento.

As principais estratégias de coordenação dos cuidados por intermédio da integração entre níveis assistenciais observados nos quatro casos coadunam-se aos encontrados por Conill \& Fausto ${ }^{5}$ para os países da América Latina. Os resultados obtidos no estudo, construídos com base na percepção de gestores, profissionais e famílias cadastradas apontaram que, nos municípios estudados, estão presentes instrumentos de integração entre níveis assistenciais, em graus distintos de implementação, indicando preocupação com a garantia de atenção integral. Foram identificadas iniciativas de articulação da Atenção Primária à Saúde aos demais serviços de saúde, fato que constitui um dos atributos que a distingue de uma concepção seletiva e focalizadora 9 . Diferenças entre avaliações de gestores, profissionais e usuários foram verificadas, assim como em outros estudos já realizados 19 .

Um ponto positivo refere-se à criação e ao fortalecimento de estruturas de regulação ao interior da SMS e nos centros de saúde. Em Araca- 
ju e Belo Horizonte, estiveram condicionados às habilitações dos sistemas municipais de saúde. Em Florianópolis e Vitória, destacou-se a capacidade de indução à organização e integração da rede potencializada pelo aumento de cobertura pela ESF, anterior à adesão do Pacto pela Saúde. Nestes municípios, a criação de estruturas de regulação foi fortemente influenciada pela expansão da atenção básica, reforçando seu caráter de porta de entrada preferencial, responsável pela coordenação entre níveis e de seu papel indutor de mudanças no modelo assistencial. Contudo, nos dois casos os processos são ainda incipientes. Embora mudanças organizacionais tenham sido implementadas ao interior da SMS, só agora se refletem nos processos de trabalho das equipes de saúde da família e no cuidado prestado aos usuários.

No caso dos municípios que optaram pelo SISREG, foi possível conhecer o tamanho real das filas de espera, monitorá-las, planejar a oferta de atenção especializada em função da demanda, definir prioridades clínicas, monitorar o índice de abstenção a consultas e exames, além de garantir maior imparcialidade no controle das agendas. Os processos de regulação da atenção especializada tradicionalmente coordenados pelas secretarias de saúde, gradativamente estão sendo incorporados à rotina dos profissionais da atenção básica, que passam a constituir a primeira instância regulatória do SUS. Tal aspecto, considerado um avanço por gestores e gerentes, trouxe também o desafio de como garantir a qualidade da regulação descentralizada.

Outro resultado positivo considerado indicador de integração da rede foi a organização dos fluxos para a atenção especializada. A maior parte dos gestores, gerentes, profissionais e famílias confirmou que a principal forma de acesso a serviços especializados ocorre por meio de referência das USF. A implantação de centros municipais de especialidades foram estratégias para aumento da oferta de consultas e exames, oferecidos em tempo e local mais oportunos, diminuindo barreiras de acesso e possibilitando maior contato entre generalistas e especialistas que passam a atuar em um mesmo território.

O investimento em TIC como a informatização dos prontuários foi uma iniciativa prócoordenação importante, ainda que em estágio inicial. O desafio parece ser integrá-lo à rede especializada e a demais prestadores, como os privados e estaduais. O desenvolvimento de instrumentos de coordenação clínica como protocolos, inclusive de acesso à atenção especializada, também representa estratégia para garantia de atenção integral, tendo como centro os serviços da rede básica.
Embora tenha sido identificado um conjunto de medidas pró-coordenação com base na implantação de estratégias organizacionais de integração da rede, alguns desafios permanecem neste campo e outros o extrapolam.

Ainda em relação à integração entre níveis assistenciais, segundo Calnan et al. 1, a literatura sugere que entre as tradicionais barreiras para a coordenação dos cuidados, destaca-se a existência de diferentes prestadores de serviços de saúde. Em Florianópolis e Vitória, a presença de prestadores estaduais cuja regulação não está sob responsabilidade do município, constitui entrave à garantia de atenção especializada, visto ainda representarem parcela importante da oferta. Assim, as unidades estaduais conformam estruturas paralelas, não integradas aos serviços especializados municipais.

No campo do cuidado hospitalar, a ausência de centrais municipais de regulação de leitos e internações, além de dificultar o acesso, impede a definição de fluxos formais, resultando em importante obstáculo à oferta de atenção integral e tornando incompleto o processo de integração da rede. O contato informal entre profissionais oportuniza acesso desigual a internações e cirurgias, nem sempre orientados por critérios de prioridade clínica. Não obstante, a ausência de mecanismos de contra-referência para a rede básica reflete-se no alto percentual de profissionais que não souberam informar o tempo de espera dos usuários para cirurgias eletivas. Situação mais favorável foi observada em relação à garantia de agendamento para maternidade, sugerindo melhorias na atenção à mulher.

Nos quatro casos, a prática sistemática de avaliação dos encaminhamentos por centro de saúde poderia melhorar a qualificação das referências e promover utilização mais racional dos recursos especializados. Esforços deveriam ser empreendidos também junto aos demais profissionais da rede quanto à necessidade de garantir a continuidade informacional, requisito para a coordenação do cuidado pela Atenção Primária à Saúde. A ausência da contra-referência sugere necessidade de maior integração entre profissionais da atenção básica e especializada.

Foi consensual também a avaliação de que, para além da organização dos fluxos e integração da rede, a garantia de atenção integral depende da suficiente oferta de procedimentos especializados. De forma diferenciada entre os municípios, nem sempre é possível garantir agendamento para média complexidade, particularmente no que se refere à rede de apoio à diagnose e terapia. A maior parte dos médicos e enfermeiros avaliou problemas decorrentes das listas de espera que impedem o acesso adequado ao cuidado 
especializado e hospitalar como o principal entrave à integração da rede de serviços de saúde (Tabela 3). Contudo, mais que a falta de fluxos ou de mecanismos de marcação e regulação de procedimentos, este e outros achados sugerem obstáculos vinculados à insuficiente oferta.

A contratação de serviços da rede privada foi uma das estratégias para suprir deficiências na oferta de atenção especializada, dificultada, segundo avaliação dos gestores e gerentes, pela remuneração oferecida pela tabela do SUS, considerada inadequada pelos prestadores. Também foi recorrente a avaliação de que não há políticas para a média complexidade promovidas pelo Ministério da Saúde. Percentual importante dos investimentos para garantir atendimento integral, que necessariamente envolve serviços especializados e hospitalares, faz parte das iniciativas e esforços do gestor municipal de acordo com os entrevistados.

\section{Resumo}

O artigo analisa o desenvolvimento de instrumentos de coordenação desde a Estratégia Saúde da Família aos demais níveis do sistema de saúde com foco em medidas pró-coordenação vinculadas à "integração entre níveis assistenciais". Os resultados obtidos em estudos de casos realizados em quatro grandes centros urbanos apontaram que estão presentes mecanismos de integração entre níveis assistenciais, indicando preocupação com a garantia de atenção integral. As principais estratégias identificadas foram: criação $e$ fortalecimento de estruturas regulatórias no interior das Secretarias Municipais de Saúde e nas unidades de saúde da família com descentralização de funções para o nível local, organização dos fluxos, prontuários eletrônicos e ampliação da oferta de serviços especializados municipais. Contudo, a falta de integração entre diferentes prestadores, a insuficiência de fluxos formais para atenção hospitalar e a ausência de políticas para média complexidade foram apontadas como entraves à garantia do cuidado integral, tornando incompleto o processo de integração da rede.

Atenção Primária à Saúde; Integração de Sistemas; Serviços de Saúde
Em síntese, estratégias semelhantes de integração da rede, com resultados diferenciados, foram encontradas nos quatro casos como investimentos em sistemas informatizados e descentralizados de regulação, monitoramento das filas de espera para a atenção especializada, aumento da oferta de serviços próprios municipais, implantação de protocolos clínicos, além dos prontuários eletrônicos em dois casos. Todavia, a ausência de regulação e de fluxos formais para a atenção hospitalar constitui importante entrave à garantia de cuidado integral, tornando incompleto o processo de integração da rede. A insuficiência na oferta de atenção especializada, agravada pela pouca integração entre prestadores estaduais e municipais, também se reflete nas dificuldades para agendamento de procedimentos especializados. Para tal, futuros estudos centrados em avaliações de cobertura da média complexidade, integração da rede na perspectiva dos profissionais que atuam na atenção especializada e constituição de fluxos para a atenção hospitalar podem trazer novas contribuições e elementos ao debate.

\section{Colaboradores}

P. F. Almeida participou da concepção, redação e análise dos dados do artigo. L. Giovanella contribuiu com a concepção e revisão crítica do artigo. M. H. M. Mendonça e S. Escorel revisaram criticamente o artigo.

\section{Agradecimentos}

Os autores agradecem aos gestores, coordenadores de Atenção Básica/ESF e demais gerentes das Secretarias Municipais de Saúde, profissionais das equipes de saúde da família e à população entrevistada pela disponibilidade em compartilhar seu conhecimento, experiência e tempo na realização das entrevistas. Ao Departamento de Atenção Básica, Secretaria de Atenção à Saúde, Ministério da Saúde e Fundo Nacional de Saúde, agradecemos os incentivos e financiamento para a realização deste estudo.

Agradecemos também o apoio do Teasdale-Corti Global Research Paternship Program, financiado pela Canadian Global Health Research Initiative, e de seus organizadores. $\mathrm{O}$ artigo expressa a visão dos autores e não necessariamente reflete a posição dos financiadores. 


\section{Referências}

1. Calnan M, Hutten J, Tiljak H. The challenge of coordination: the role of primary care professional in promoting integration across the interface. In: Saltman RS, Rico A, Boerma WGW, editors. Primary care in the driver's seat? Organizational reform in European primary care. Berkshire: Open University Press; 2007. p. 85-104

2. Gérvas J, Rico A. La coordinación en el sistema sanitario y su mejora a través de las reformas europeas de la Atención Primaria. SEMERGEN 2005; 31:418-23.

3. Hofmarcher MM, Oxley H, Rusticelli E. Improved health system performance through better care coordination. Paris: Organisation for Economic Co-operation and Development; 2007.

4. Giovanella L. A atenção primária à saúde nos países da União Européia: configurações e reformas organizacionais na década de 1990. Cad Saúde Pública 2006; 22:951-63.

5. Conill E, Fausto M. Análisis de la problemática de la integración de la APS en el contexto actual: causas que inciden en la fragmentación de servicios y sus efectos en la cohesión social. Rio de Janeiro: EuroSocial Salud; 2007.

6. Núñez RT, Lorenzo IV, Naverrete ML. La coordinación entre niveles asistenciales: una sistematización de sus instrumentos y medidas. Gac Sanit 2006; 20:485-95.

7. Boerma WGW. Coordination and integration in European primary care. In: Saltman RS, Rico A, Boerma WGW, editors. Primary care in the driver's seat? Organizational reform in European primary care. Berkshire: Open University Press; 2007. p. 3-21.

8. Hartz ZMA, Contandriopoulos A-P. Integralidade da atenção e integração de serviços de saúde: desafios para avaliar a implantação de um "sistema sem muros”. Cad Saúde Pública 2004; 20 Suppl 2:S331-6.

9. Word Health Organization. Primary health care. Now more than ever. The World Health Report 2008. Geneva: Word Health Organization; 2008.

10. Giovanella L, Escorel S, Mendonça MHM, coordenadores. Estudos de caso sobre a implementação da Estratégia Saúde da Família em grandes centros urbanos. Relatório final. Brasília: Ministério da Saúde; 2009.

11. Watson DE, Broemeling AM, Reid RJ, Black C. A results-based logic model for primary health care: laying an evidence-based foundation to guide performance measurement, monitoring and evaluation. Vancouver: Centre for Health Services and Policy Research, University of British Columbia; 2004.
12. Ojeda JJ, Freire JM, Gérvas J. La coordinación entre atención primaria y especializada: reforma del sistema sanitario o reforma del ejercicio profesional. Revista de Administración Sanitaria 2006; 4:357-82.

13. Almeida C, Macinko J. Validação de uma metodologia de avaliação rápida das características organizacionais e do desempenho dos serviços de atenção básica do Sistema Único de Saúde (SUS) em nível local. Brasília: Organização Pan-Americana da Saúde/Ministério da Saúde; 2006. (Série Técnica Desenvolvimento de Sistemas e Serviços de Saúde, 10).

14. Giovanella L, Escorel S, Mendonça MHM. Porta de entrada pela atenção básica? Integração do PSF à rede de serviços de saúde. Saúde Debate 2003; 27:278-89

15. Escorel S, Giovanella L, Mendonça MHM, Senna MCM. Avaliação da implementação do Programa Saúde da Família em dez grandes centros urbanos: síntese dos principais resultados. 2a Ed. Brasília: Ministério da Saúde; 2005.

16. Departamento de Atenção Básica, Secretaria de Atenção à Saúde, Ministério de Saúde. Avaliação para melhoria da qualidade da Equipe de Saúde da Família. Brasília: Ministério da Saúde; 2005. (Série B. Textos Básicos de Saúde).

17. Viana ALA, Rocha JSY, Elias PE, Ibañez N, Novaes MHD. Modelos de atenção básica nos grandes municípios paulistas: efetividade, eficácia, sustentabilidade e governabilidade. Ciênc Saúde Coletiva 2006; 11:577-606.

18. Elias PE, Ferreira CW, Alves MCG, Cohn A, Kishima V, Escrivão Júnior A, et al. Atenção Básica em Saúde: comparação entre PSF e UBS por extrato de exclusão social no município de São Paulo. Ciênc Saúde Coletiva 2006; 11:633-41.

19. Ibañez N, Rocha JSY, Castro PC, Ribeiro MCSA, Forster AC, Novaes MHD, et al. Avaliação do desempenho da atenção básica no Estado de São Paulo. Ciênc Saúde Coletiva 2006; 11:683-703.

20. Facchini LA, Piccini RX, Tomasi E, Thumé E, Silveira DS, Siqueira FV, et al. Desempenho do PSF no Sul e no Nordeste do Brasil: avaliação institucional e epidemiológica da Atenção Básica à Saúde. Ciênc Saúde Coletiva 2006; 11:669-81.

21. Ministério da Saúde/Fundação Oswaldo Cruz. Pesquisa Mundial de Saúde/Municípios do Brasil/ Atenção Básica. Rio de Janeiro: Ministério da Saúde/Fundação Oswaldo Cruz; 2005.

22. Starfield B. Atenção Primária: equilíbrio entre necessidades de saúde, serviços e tecnologia. Brasília: Organização das Nações Unidas para a Educação, a Ciência e a Cultura/Ministério da Saúde; 2002.

Recebido em 24/Mar/2009

Versão final reapresentada em 31/Out/2009

Aprovado em 10/Nov/2009 